University of Rhode Island

DigitalCommons@URI

Mechanical, Industrial \& Systems Engineering

Faculty Publications

Mechanical, Industrial \& Systems Engineering

2013

\title{
Temperature measurements of heated microcantilevers using scanning thermoreflectance microscopy
}

Joohyun Kim

Sunwoo Han

Timothy Walsh

University of Rhode Island

Keunhan Park

University of Rhode Island

Bong Jae Lee

See next page for additional authors

Follow this and additional works at: https://digitalcommons.uri.edu/mcise_facpubs

Terms of Use

All rights reserved under copyright.

\section{Citation/Publisher Attribution}

Kim, J., Han, S., Walsh, T., Park, K., Lee, B. J., King, W. P., \& Lee, J. (2013). Temperature measurements of heated microcantilevers using scanning thermoreflectance microscopy. Rev. Sci. Instrum., 84(3), 034903. doi: 10.1063/1.4797621

Available at: https://doi.org/10.1063/1.4797621

This Article is brought to you for free and open access by the Mechanical, Industrial \& Systems Engineering at DigitalCommons@URI. It has been accepted for inclusion in Mechanical, Industrial \& Systems Engineering Faculty Publications by an authorized administrator of DigitalCommons@URI. For more information, please contact digitalcommons-group@uri.edu. 


\section{Authors}

Joohyun Kim, Sunwoo Han, Timothy Walsh, Keunhan Park, Bong Jae Lee, William P. King, and Jungchul Lee

This article is available at DigitalCommons@URI: https://digitalcommons.uri.edu/mcise_facpubs/29 


\section{Temperature measurements of heated microcantilevers using scanning thermoreflectance microscopy}

Cite as: Rev. Sci. Instrum. 84, 034903 (2013); https://doi.org/10.1063/1.4797621

Submitted: 22 December 2012 . Accepted: 10 March 2013 . Published Online: 26 March 2013

Joohyun Kim, Sunwoo Han, Timothy Walsh, Keunhan Park, Bong Jae Lee, William P. King, and Jungchul Lee

\section{ARTICLES YOU MAY BE INTERESTED IN}

Note: Simultaneous determination of local temperature and thickness of heated cantilevers using two-wavelength thermoreflectance

Review of Scientific Instruments 85, 036109 (2014); https://doi.org/10.1063/1.4869079

Nanoscale thermal transport

Journal of Applied Physics 93, 793 (2003); https://doi.org/10.1063/1.1524305

Thermal conductivity measurement from 30 to $750 \mathrm{~K}$ : the $3 \omega$ method

Review of Scientific Instruments 61, 802 (1990); https://doi.org/10.1063/1.1141498

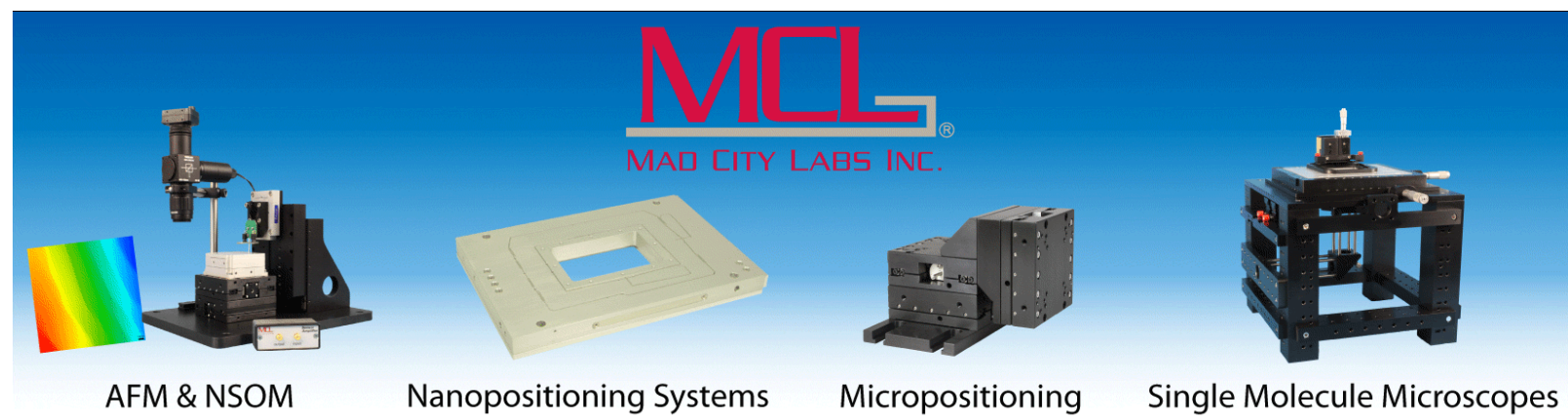




\title{
Temperature measurements of heated microcantilevers using scanning thermoreflectance microscopy
}

\author{
Joohyun Kim, ${ }^{1}$ Sunwoo Han, ${ }^{2}$ Timothy Walsh, ${ }^{3}$ Keunhan Park, ${ }^{3}$ Bong Jae Lee, ${ }^{2}$ \\ William P. King, ${ }^{4}$ and Jungchul Lee ${ }^{1, a)}$ \\ ${ }^{1}$ Department of Mechanical Engineering, Sogang University, Seoul 121-742, South Korea \\ ${ }^{2}$ Department of Mechanical Engineering, Korea Advanced Institute of Science and Technology, \\ Daejeon 305-701, South Korea \\ ${ }^{3}$ Department of Mechanical, Industrial, and Systems Engineering, University of Rhode Island, Kingston, \\ Rhode Island 02881, USA \\ ${ }^{4}$ Department of Mechanical Science and Engineering, University of Illinois at Urbana-Champaign, Urbana, \\ Illinois 61801, USA
}

(Received 22 December 2012; accepted 10 March 2013; published online 26 March 2013)

\begin{abstract}
We report the development of scanning thermoreflectance thermometry and its application for steady and dynamic temperature measurement of a heated microcantilever. The local thermoreflectance signal of the heated microcantilever was calibrated to temperature while the cantilever was under steady and periodic heating operation. The temperature resolution of our approach is $0.6 \mathrm{~K}$, and the spatial resolution is $2 \mu \mathrm{m}$, which are comparable to micro-Raman thermometry. However, the temporal resolution of our approach is about $10 \mu \mathrm{sec}$, which is significantly faster than micro-Raman thermometry. When the heated microcantilever is periodically heated with frequency up to $100 \mathrm{kHz}$, we can measure both the in-phase and out-of-phase components of the temperature oscillation. For increasing heating frequency, the measured cantilever AC temperature distribution tends to be confined in the vicinity of the heater region and becomes increasingly out of phase with the driving signal. These results compare well with finite element simulations. (C) 2013 American Institute of Physics. [http://dx.doi.org/10.1063/1.4797621]
\end{abstract}

\section{INTRODUCTION}

A heated microcantilever is a functional atomic force microscope cantilever that has an integrated microscale heater near the scanning tip. ${ }^{1,2}$ With highly localized heating capability, heated microcantilevers are actively used for nanoscale thermal manufacturing ${ }^{3-7}$ and metrology on various materials, including polymers, ${ }^{8-12}$ explosives, ${ }^{4}$ carbon nanotubes, ${ }^{13}$ and graphene. ${ }^{14}$ Periodic heating (or AC) operation of heated microcantilevers is a promising technique that offers unique metrology applications, including thermomechanical actuation, ${ }^{15,16}$ thermal topographic imaging, ${ }^{17}$ and local thermal analysis. ${ }^{18}$ However, AC thermal characterization of heated microcantilevers to date mostly relies on theoretical modeling and indirect measurement. ${ }^{19-21}$ Although transient temperature measurements of a heated microcantilever was performed by acquiring temperature-dependent reflectance, ${ }^{22}$ measurements were limited to the pulsed operation in time domain at a fixed point and detailed discussion on temperature, spatial, and temporal resolutions was not provided. Therefore, it is crucial to measure the temperature distribution of heated microcantilevers during $\mathrm{AC}$ operation.

As a routine calibration procedure, heated microcantilevers have been electrothermally characterized using micro-Raman spectroscopy. ${ }^{1,23,24}$ While micro-Raman spectroscopy provides $\sim 1 \mu \mathrm{m}$ spatial and $\pm 4{ }^{\circ} \mathrm{C}$ temperature resolutions, ${ }^{25}$ it requires $\sim 10 \mathrm{sec}$ of integration time to col-

a) Author to whom correspondence should be addressed. Electronic mail: jayclee@ sogang.ac.kr lect very weak Raman scattering signals. Due to this limited temporal resolution, the micro-Raman spectroscopy is more suitable for DC characterization and cannot be directly applied to the AC thermal characterization of the heated microcantilever. Since thermal time constants of the heated microcantilever range between $100 \mu \mathrm{sec}$ and $1 \mathrm{msec},{ }^{1,20}$ electrical and thermal behaviors of the heated microcantilever strongly depend on the operating frequency. There is a need for a microthermometry technique with high temporal resolution suitable for measuring heated microcantilevers.

This paper presents scanning thermoreflectance microscopy ${ }^{26-28}$ for steady and transient thermal characterizations of microdevices. In thermoreflectance measurements, the reflected signal of an incident light source is collected and analyzed ${ }^{29,30}$ while a sample of interest is being heated. When compared to the Raman spectroscopy, acquiring the optical reflection greatly simplifies measurement procedure and setup, and can improve the temporal resolution significantly.

\section{EXPERIMENTAL SETUP}

The scanning thermoreflectance microscope shown in Fig. 1(a) was constructed based on an industrial microcope (Nikon, LV100) with an optional dual port and several optical components including a polarized diode laser (Coherent, ULN $\lambda=633 \mathrm{~nm}, \mathrm{P}=5 \mathrm{~mW}$ ), a $5 \times$ beam expander (Thorlabs, BE05M-B), a cube beam splitter (Thorlabs, CM1BS013), a silicon detector (Thorlabs, DET100A/M), and a CCD camera (Imaging Source, DFK31BF03). Dual optical 
(a)

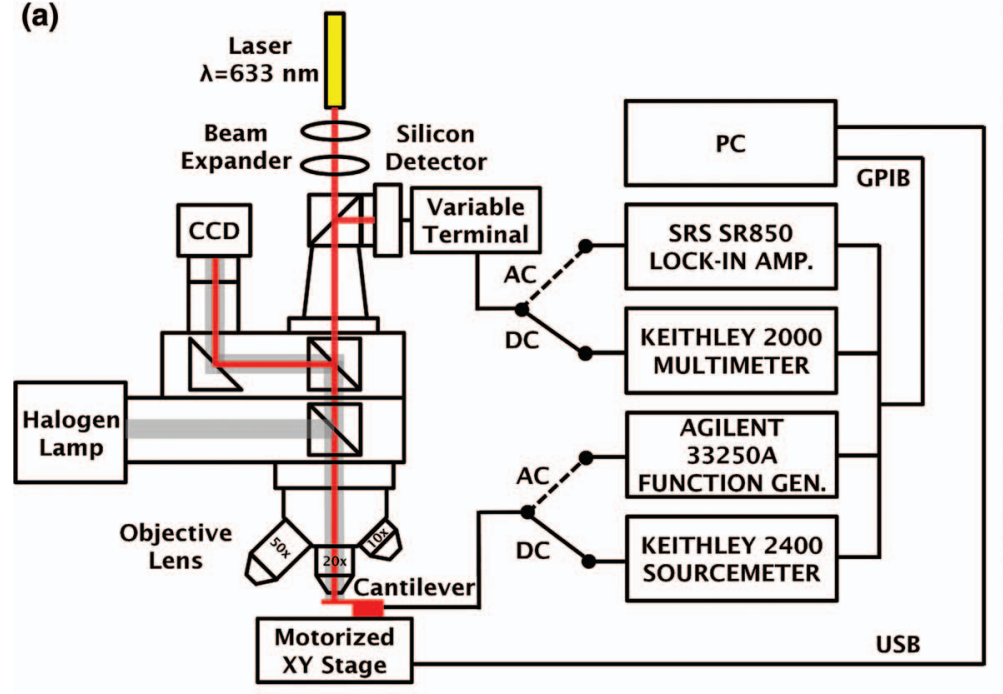

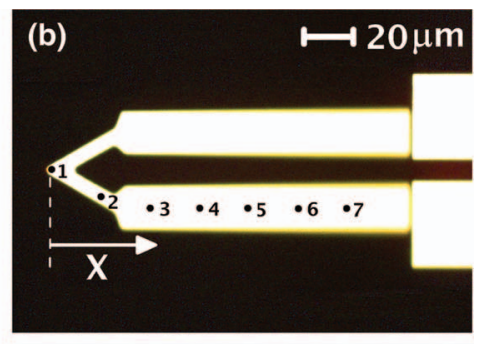

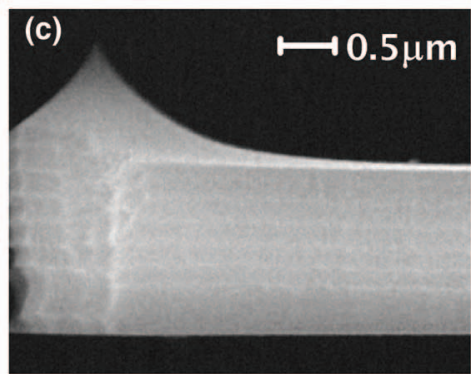

FIG. 1. (a) Experimental setup for thermoreflectance measurements of a heated microcantilever under DC or AC operation. (b) An optical micrograph (top-view) of the heated microcantilever. Seven dots with numbering indicate specific positions for thermoreflectance measurements. (c) A scanning electron micrograph (side-view) of the heated microcantilever showing the thickness around the scanning tip.

ports of the microscope were occupied by the CCD camera and the laser assembly, including the laser, the beam expander, the beam splitter, and the silicon detector. The polarized laser was expanded and focused on the heated microcantilever shown in Figs. 1(b) and 1(c) through a $50 \times$ microscope objective $(\mathrm{NA}=0.8)$. The reflected beam traveled back and was incident on the silicon detector. Photocurrent generated in the silicon detector was converted to voltage via a variable terminal (Thorlabs, VT1). The CCD camera mainly aided the laser alignement onto the heated microcantilever. The laser focal spot was approximately $1.5 \mu \mathrm{m}$ in diameter based on the theoretical estimation from our optical configurations. Note that this spot size is smaller than the resolution of the microstage used for scanning $(\sim 2 \mu \mathrm{m})$. Thus, the spatial resolution of our thermoreflectance setup is limited by the microstage rather than the laser focal spot.

The heated microcantilever connected with a current limiting series resistor $(6.8 \mathrm{k} \Omega)$ was mounted on top of an $\mathrm{x}-\mathrm{y}$ motorized stage (Thorlabs, two PT1/M-Z8 are stacked). The scanning electron micrograph shown in Fig. 1(c) provides the estimation of the thicknesses around the scanning tip. For DC operation, the heated microcantilever connected with the resistor was powered with a sourcemeter (Keithley, 2400) while the sourcemeter measured the current and a multimeter (Keithley, 2000) measured the voltage drop across the resistor. For AC operation, a function generator (Agilent, 33250A) was used to modulate the power dissipation in the heated microcantilever and a lock-in amplitfier (Stanford Rearch Systems, SR850) was used to measure the in-phase and out-of-phase components of the thermoreflectance signal. The position of the microcantilever was fixed for single point measurements and was raster-scanned for mapping. All instruments connected to a computer via GPIB or USB enabled fully automated measurements.

\section{RESULTS AND DISCUSSION}

\section{A. Preliminary measurements}

While the heated microcantilever was electrically characterized, the thermoreflectance signal from the tip (i.e., position 1 in Fig. 1(b)) was simultaneously measured. Figure 2(a) shows the cantilever resistance and thermoreflectance signal from the tip as a function of the cantilever power dissipation. As the cantilever power dissipation increases, the cantilever electrical resistance exhibits a nonlinear increase and characteristic thermal-runaway at $8 \mathrm{~mW} .{ }^{1,31}$ The thermoreflectance signal from the tip exhibits an oscillatory pattern having the local minimum and maximum values. By inspecting several heated microcantilevers from the same batch, we found that the oscillatory thermoreflectance signals are due to interference of multiply reflected laser light inside the cantilever, hence strongly depend on the thickness of the heated microcantilever. Figure 2(b) shows the tip temperature measured with the micro-Raman spectroscopy as a function of the cantilever power dissipation, which is consistent with results from previous publications. ${ }^{1}$ Raman measurements were repeated at several points along the heated microcantilever and later used for calibrating thermoreflectance measurements.

Figure 2(c) shows thermoreflectance signals measured at the tip position (position A) and $2 \mu \mathrm{m}$ offset from the tip (position B) as a function of temperature. Although all signals exhibit an oscillatory behavior, their local minimum and maximum points are considerably different. As the measurement point offsets slightly from the tip, both the local minimum and maximum points shift to higher temperatures. When considering the thickness of $\sim 2.33 \mu \mathrm{m}$ and $\sim 1.83 \mu \mathrm{m}$ at points $\mathrm{A}$ and $\mathrm{B}$, respectively, as estimated from the scanning electron micrograph in Fig. 1(c), the local thickness variation of $\sim 500 \mathrm{~nm}$ significantly alter the thermoreflectance signal. Figure 2(d) shows theoretically calculated reflectance of 1.83 and $2.33-\mu \mathrm{m}$-thick silicon at $633 \mathrm{~nm}$ in wavelength as a 
(a)

(b)

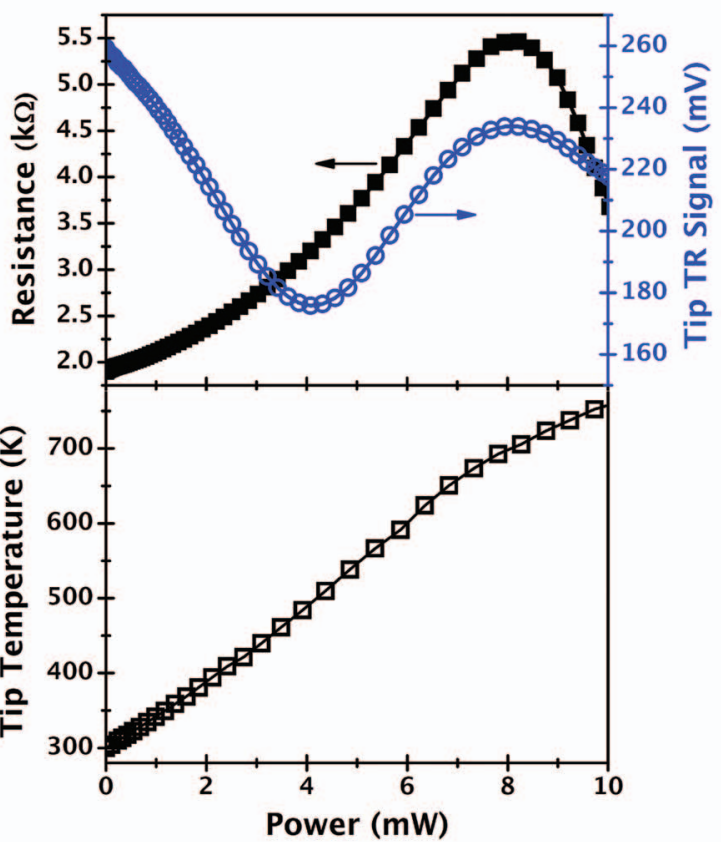

(c)

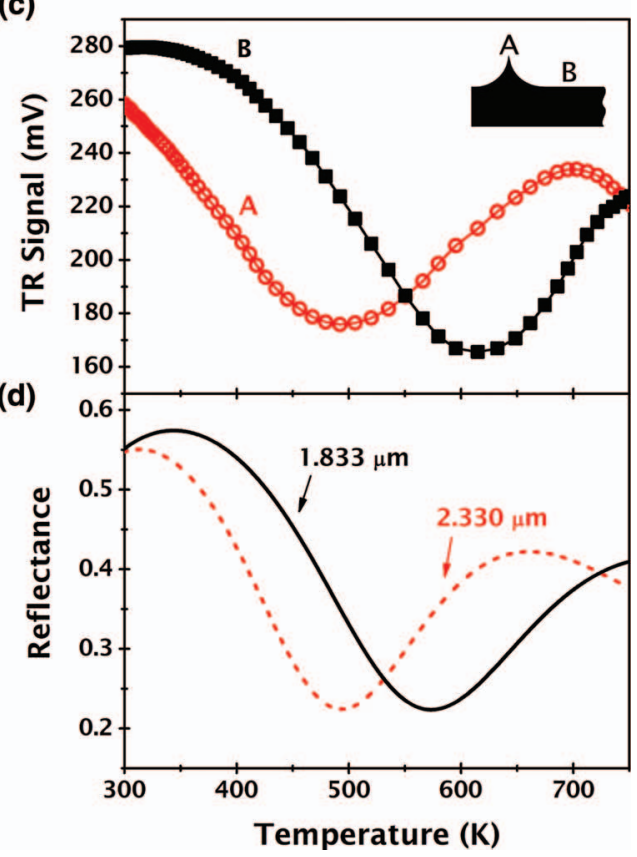

FIG. 2. (a) Resistance of the heated microcantilever (left y-axis) and thermoreflectance signal at the tip (right y-axis) as a function of the power dissipation in the heated microcantilever. (b) Temperature at the tip measured with micro Raman thermometry as a function of the power dissipation. (c) Thermoreflectance signal measured at the tip (position A) and near the tip (position B) as a function of temperature. (d) Theoretical reflectance of 1.833 and $2.330 \mu$ m thick silicon for the wavelength of $633 \mathrm{~nm}$ as a function of temperature that strongly depends on the thickness. Thicknesses of 1.833 and $2.330 \mu \mathrm{m}$ agree with estimation from the scanning electron micrograph shown in Fig. 1(c).

function of temperature. In the calculation, we have employed the model by Jellison and Modine ${ }^{32}$ for the optical constants of silicon as a function of temperature. Notice that at $633 \mathrm{~nm}$ free carriers from ionized-donors have negligible effects on the optical constant because the bandgap contribution is dominant. With the temperature-dependent optical constants, the reflectance of a thin silicon layer has been calculated based on the thin-film optics. ${ }^{33}$ As shown in Fig. 2(d), the theoretical reflectance captures the essential features of the measured value, verifying that the thermoreflectance is very sensitive to the local thickness variation and that the sharp cantilever tip does not significantly alter the thermoreflectance signal.

For the thickness range of heated microcantilevers used, the absorptance of silicon at $633 \mathrm{~nm}$ wavelength ranges from 0.3 to 0.6 depending on the temperature. During our thermoreflectance measurements, the laser intensity focused on the heated microcantilever was measured to be approximately $400 \mu \mathrm{W}$. Therefore, the absorbed optical power by the microcantilever could be estimated as 120-240 $\mu \mathrm{W}$. If the absorbed optical power is fully dissipated as heat, the temperature rise due to the laser heating is estimated to be $10 \mathrm{~K}$ at most when the laser was focused near the free end. Laser heating effect would be mitigated if the laser focus is away from the free end since heating becomes less efficient due to the heat conduction through the cantilever legs.

\section{B. DC thermoreflectance measurements}

In order to measure the temperature distribution of the heated microcantilever, thermoreflectance mapping was performed using the $x-y$ motorized stage. Figure 3(a) shows thermoreflectance contour images at DC power dissipations of $0,3.9,7.1$, and $9.9 \mathrm{~mW}$ with the scanning step of $2 \mu \mathrm{m}$. The measured thermoreflectance shows the most significant variation near the free end, while it is insensitive to the input power near the anchor. This is due to temperature-sensitive thermoreflectance signals (refer to Fig. 2), and the largest temperature gradient is expected near the free end of the heated microcantilever. Figure 3(b) shows the temperature distributions along the heated microcantilever for different DC power dissipations, converted from the thermoreflectance using the micro-Raman calibration result shown in Fig. 2(b). Temperatures are highest at the free end and drastically decrease along the cantilever, suggesting that most of heat dissipates at the heater region due to its low doping concentration of $\sim 10^{17} / \mathrm{cm}^{3}$, which is three orders of magnitude lower than the leg region. More temperature values were measured at the seven discrete points with increasing the cantilever power and shown in Fig. 3(c). While local temperatures increase with increasing power dissipation at each position, the free end temperature exhibits the highest slope at a given power and reaches $\sim 750 \mathrm{~K}$ at $10 \mathrm{~mW}$.

\section{AC thermoreflectance measurements}

The AC power dissipation of the heated microcantilever was modulated with a function generator and AC thermoreflectance signals were measured with the lock-in technique at various modulation frequencies. Figure 4(a) shows rootmean-squared (RMS) thermoreflectance oscillation contours (or AC thermoreflectance) when the total input voltage is $17 \mathrm{~V}_{\mathrm{pp}}$ at $20 \mathrm{~Hz}, 200 \mathrm{~Hz}, 2 \mathrm{kHz}$, and $20 \mathrm{kHz}$. At low 
(a)
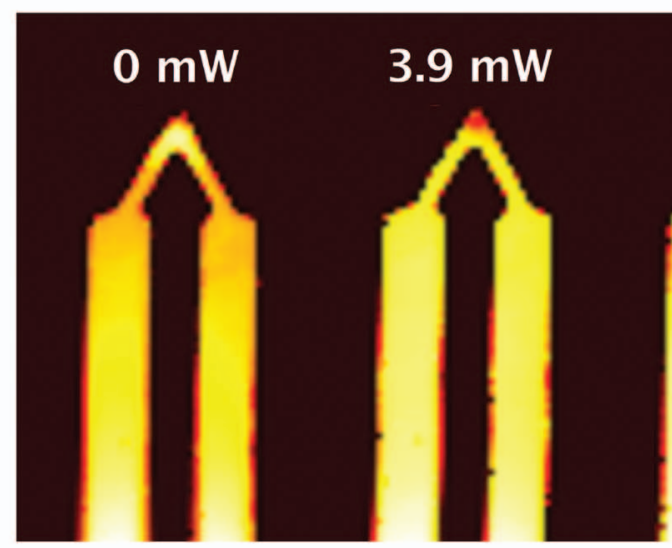

(b)

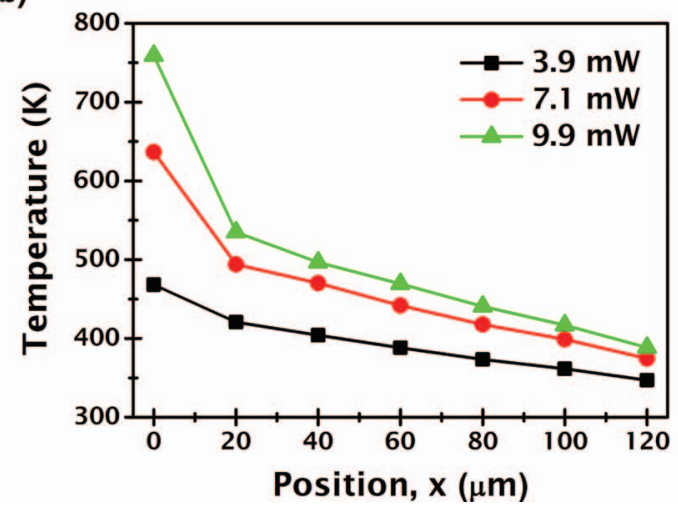

TR ( $m$ V)

296

264

231

198

165

(c)

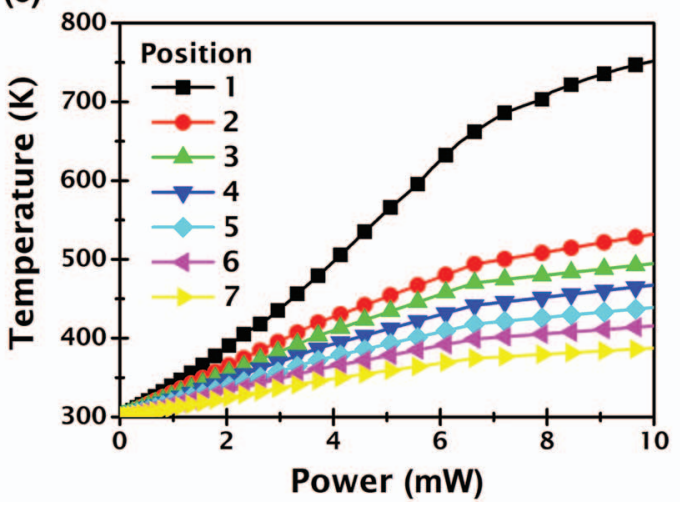

FIG. 3. (a) Thermoreflectance map of the heated microcantilever during DC operation at 0, 3.9, 7.1, and 9.9 mW. (b) Temperature as a function of the position at $3.9,7.1$, and $9.9 \mathrm{~mW}$. (c) Temperature as a function of the power dissipation along the heated microcantilever (1-7). Position, $\mathrm{x}$, is referenced to the free end of the heated microcantilever (see Fig. 1(b)).

frequencies, the tip temperature oscillates along the DC curve shown in Fig. 2(b). The $17 \mathrm{~V}_{\mathrm{pp}}$ input bias gives rise to the averaged AC cantilever power dissipation at $1.03 \mathrm{~mW}$ (or power oscillation from 0 to $2.05 \mathrm{~mW}$ at low frequencies). In such condition, local thermoreflectance signals mostly show monotonic variation with power (or temperature), as shown in Figs. 2(a) and 2(c), allowing unambiguous temperature conversion from the thermoreflectance signal. AC thermoreflectance maps are similar for 20 and $200 \mathrm{~Hz}$ modulations, indicating that at low frequencies the heated microcantilever can fully respond to the power oscillation. The color map is brighter around the tip than the neighboring area because a thicker position has larger thermoreflectance change for a given power. As the modulation frequency increases beyond $200 \mathrm{~Hz}$, the amplitude of the AC thermoreflectance signal decreases and becomes restricted to the heater region. In case of $20 \mathrm{kHz}$, the thermoreflectance signal is significantly reduced. All four AC thermoreflectance contours in Fig. 4(a) use an identical color scale bar for comparison. Figures 4(b) and 4(c) show DC-offset and AC temperature distributions along the cantilever. In Fig. 4(b), the DC-offset temperature distribution is plotted only for $20 \mathrm{~Hz}$ since DC temperatures are nearly independent of the modulation frequency. As shown in Fig. 4(b), the DC-offset temperature is highest at the tip position and drastically decreases along the cantilever.
In the AC thermoreflectance measurement, the temporal resolution of the thermoreflectance thermometry is about $10 \mu \mathrm{sec}$, which is about six orders of magnitude shorter than the typical integration time of micro-Raman thermometry. Moreover, the temporal resolution of the current thermoreflectance thermometry is not the physical limitation, but limited by the maximum frequency range of the lock-in amplifier $(102.4 \mathrm{kHz})$. It could be further improved with a faster lockin amplifier if the time constant of a device under test becomes smaller. Another noteworthy feature of Fig. 4(a) is the presence of a dark, disconnected thermoreflectance region at $\sim 8 \mu \mathrm{m}$ away from the free end regardless of the modulation frequency. The supplementary material ${ }^{34}$ contains additional details of this interesting feature.

Figure 5 shows finite element simulation results of the AC temperature distribution along the heated microcantilever for comparison with the measurement. The frequency-domain finite element analysis was developed using the COMSOL Multiphysics commercial package. The in-phase and out-ofphase temperature oscillations at the heating frequency (or $2 f$, where $f$ is the modulation frequency) were computed in the frequency domain under the steady periodic heating condition. All geometric parameters, including the cantilever thickness, were identical to the microcantilever used in the experiment. Doping concentrations at the heater and leg regions were assumed to be uniform across the cantilever thickness 
(a)

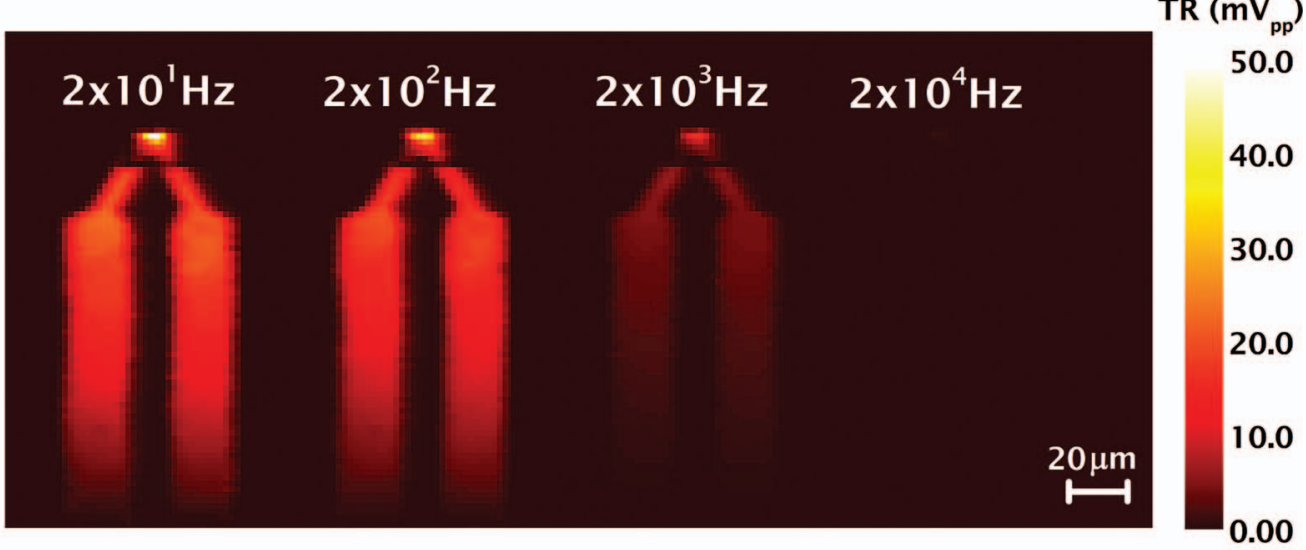

(b)

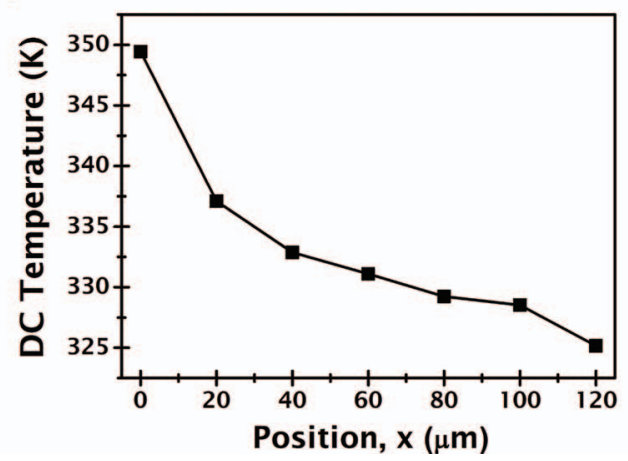

(c)

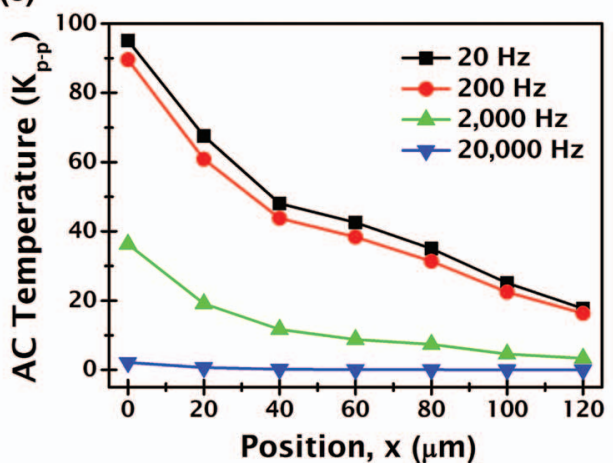

FIG. 4. (a) Measured thermoreflectance map of the heated microcantilever during AC operation at $20 \mathrm{~Hz}, 200 \mathrm{~Hz}, 2 \mathrm{kHz}$, and $20 \mathrm{kHz}$ modulation with $17 \mathrm{~V}$ pp total excitation. (b) DC temperature as a function of the position which is independent of the modulation frequency. (c) AC temperature as a function of the position at each modulation frequency.

(a)

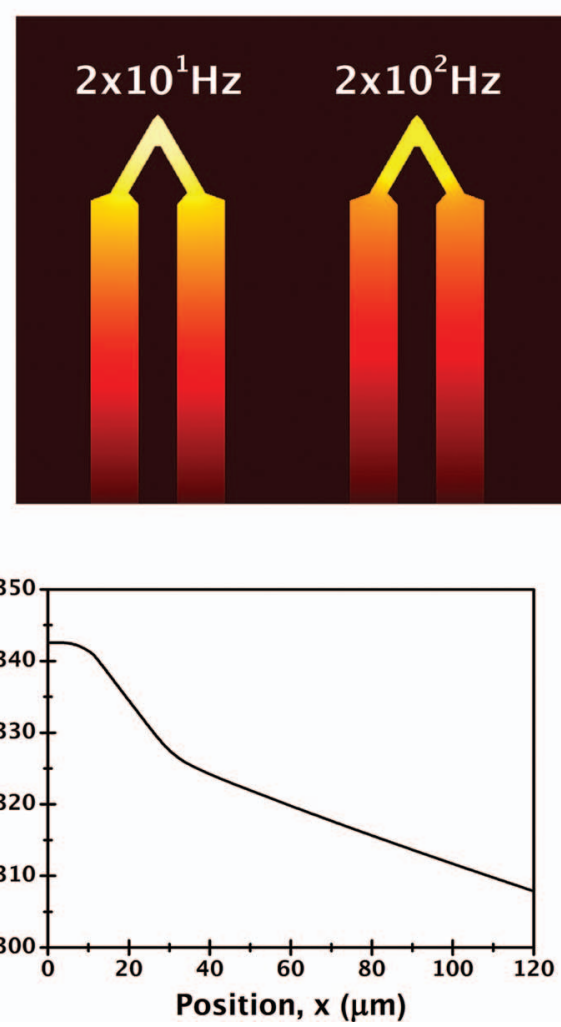

Temp. $\left(\mathrm{K}_{\mathrm{pp}}\right)$

120.0

100.0

80.0

60.0

40.0

20.0

0.0

(c)

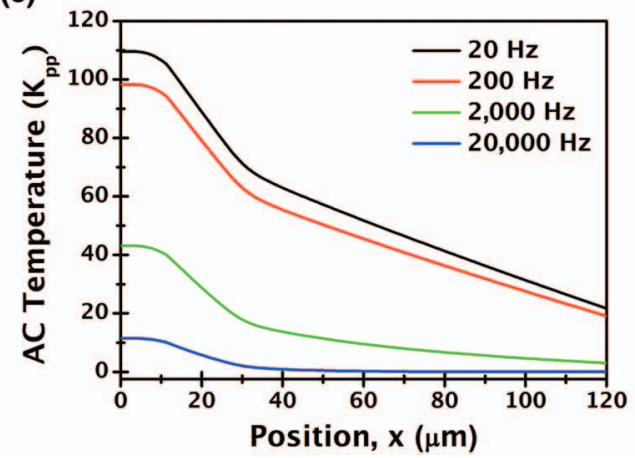

FIG. 5. (a) Simulated temperature map of the heated microcantilever during AC operation at $20 \mathrm{~Hz}, 200 \mathrm{~Hz}, 2 \mathrm{kHz}$, and $20 \mathrm{kHz}$ modulation with $17 \mathrm{~V}$ pp total excitation. (b) DC temperature as a function of the position which is independent of the modulation frequency. (c) AC temperature as a function of the position at each modulation frequency. 
(a)

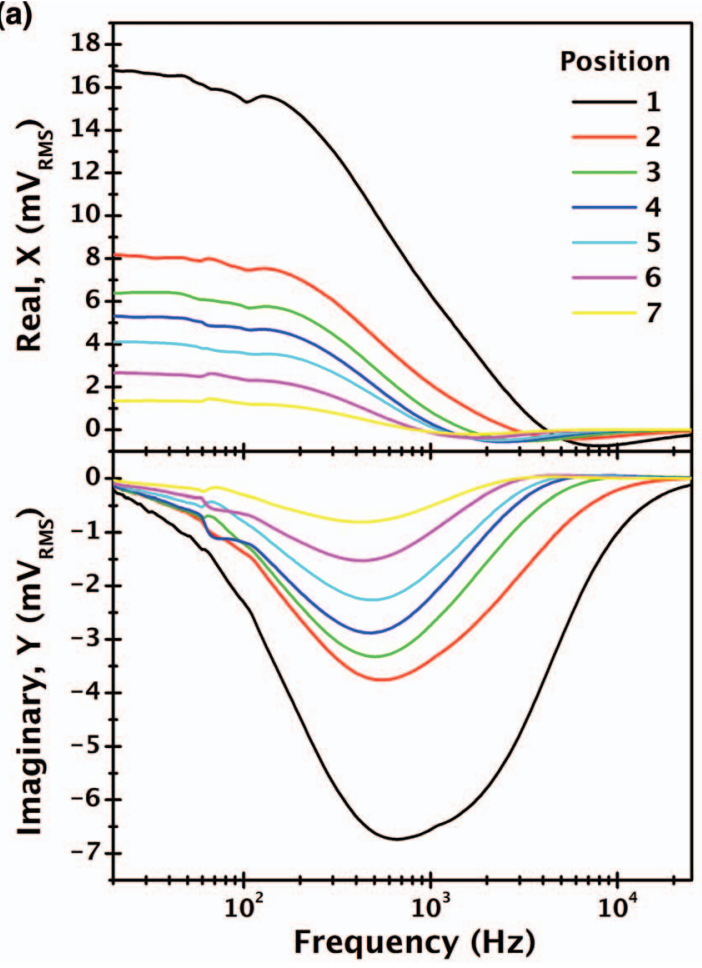

(b)

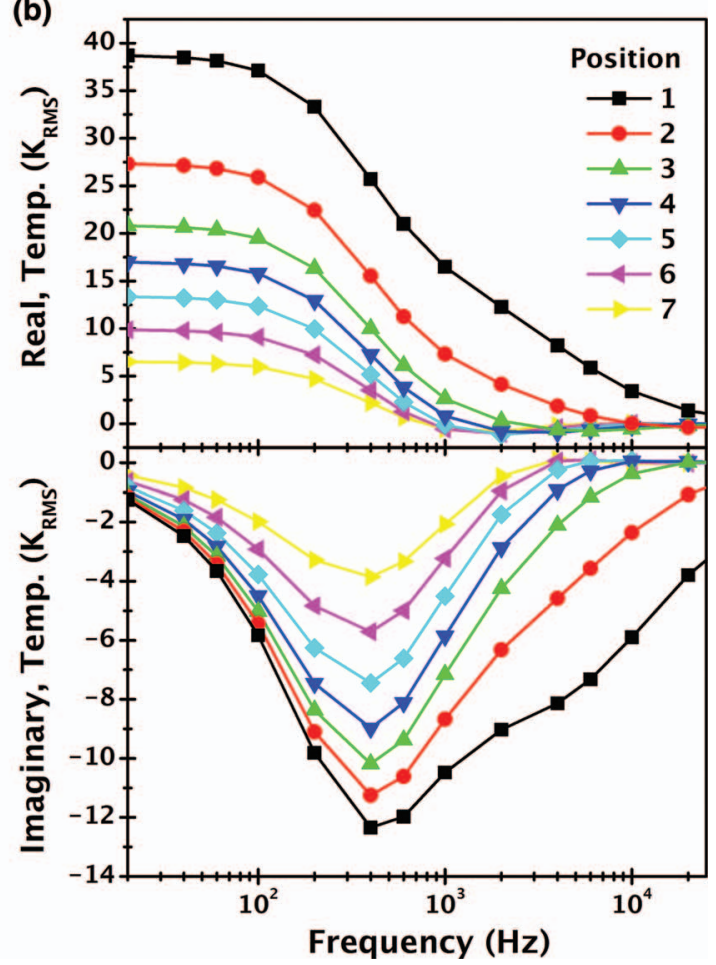

FIG. 6. Real (in-phase) and imaginary (out-of-phase) parts of (a) measured thermoreflectance signals and (b) simulated temperatures as a function of the modulation frequency at various positions along the heated microcantilever.

and determined to be $4 \times 10^{17} / \mathrm{cm}^{3}$ and $1 \times 10^{20} / \mathrm{cm}^{3}$, respectively, by computing the slope of the DC resistance versus the tip temperature and comparing with the measurement. Under the periodic heating with the same averaged power as the experiment (i.e., $1.03 \mathrm{~mW}$ ), Fig. 5 shows a very similar result to the measurement: as the frequency increases, the AC temperature shrinks to the heater region while its amplitude decreases. This temperature constriction is because the modulation frequency becomes much faster than the thermal time constant of the heated microcantilever. However, the calculation results slightly overpredict the measured temperature. When calculating the $\mathrm{AC}$ power dissipation, higher harmonic terms in temperature oscillation beyond $2 f$ were not considered by assuming the cantilever resistance to be constant at the DC-offset value. While this approximation is valid for a small AC current input, it may cause the overprediction of temperature when the cantilever oscillation becomes sufficiently large. ${ }^{15}$ Another reason might come from the electrical resistivity model of doped silicon. The carrier mobility mode $^{35}$ and ionization model ${ }^{36}$ used for this calculation are valid for $10^{15}-10^{20} \mathrm{~cm}^{-3}$ phosphorus-doping concentrations and for $250-400 \mathrm{~K}$ with $\sim 3 \%$ accuracy. Since the calculation condition slightly exceeds the valid range of the model, this may cause additional computation inaccuracy.

In order to fully understand the cantilever $\mathrm{AC}$ heating behaviors, Fig. 6 compares real and imaginary components of the measured thermoreflectance signals and the calculated temperatures at various positions of the cantilever as a function of the modulation frequency. At low frequencies, the in-phase temperature oscillation is dominant over the out-ofphase counterpart, suggesting that the cantilever temperature responds to the periodic power dissipation with almost no phase lag. However, as the frequency increases over $\sim 200 \mathrm{~Hz}$, the temperature oscillation becomes more out of phase due to the fast power oscillation exceeding the thermal time constant of the cantilever. This also explains the decrease of the temperature oscillation amplitude shown in Fig. 4 at higher frequencies. Thermal cut-off frequency, the inverse of the thermal time constant, thus exists at $\sim 1 \mathrm{kHz}$ for the tested cantilever.

The dynamic thermal responses of the heated microcantilever are further investigated by fast Fourier transforming the AC thermoreflectance signal based on the Hanning window function. Figure 7(a) shows RMS thermoreflectance spectra as a function of the normalized frequency with $17 \mathrm{~V}_{\mathrm{pp}}$ input voltage. The thermoreflectance spectra depend on the modulation frequency and have even harmonics only, indicating that the thermoreflectance signal is solely due to the periodic heating of the cantilever oscillating at even harmonics. At $20 \mathrm{~Hz}$, even harmonics of $2 f, 4 f$, and $6 f$ are clearly observed. The generation of higher harmonics is attributable to the nonlinearity of the cantilever resistance..$^{20}$ As the modulation frequency increases and exceeds the thermal cut-off frequency, $4 f$ and $6 f$ components are greatly suppressed. Figure 7(b) compares the amplitude ratio of $2 f$ to $4 f$ as a function of the modulation frequency. The amplitude ratio experiences a gradual increase until the cut-off frequency of $\sim 1 \mathrm{kHz}$, but its slope increases drastically as the frequency further increases.

\section{Thermoreflectance resolution}

Figure 8 shows the resolution of the thermoreflectance signals based on Allan variance analysis as a function of the 
(a)

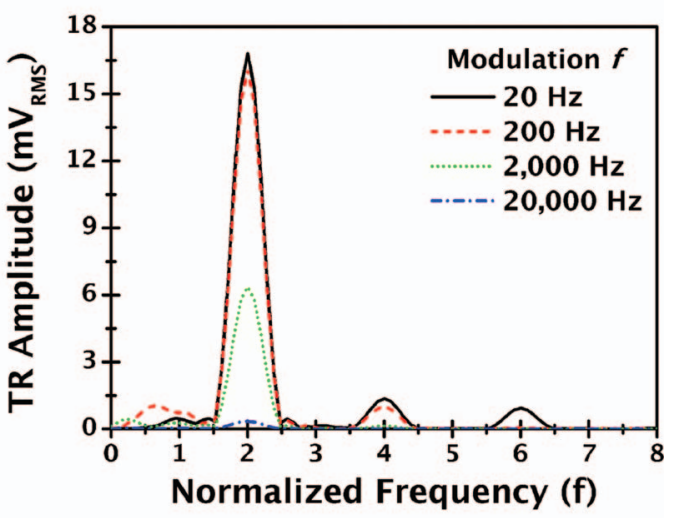

(b)

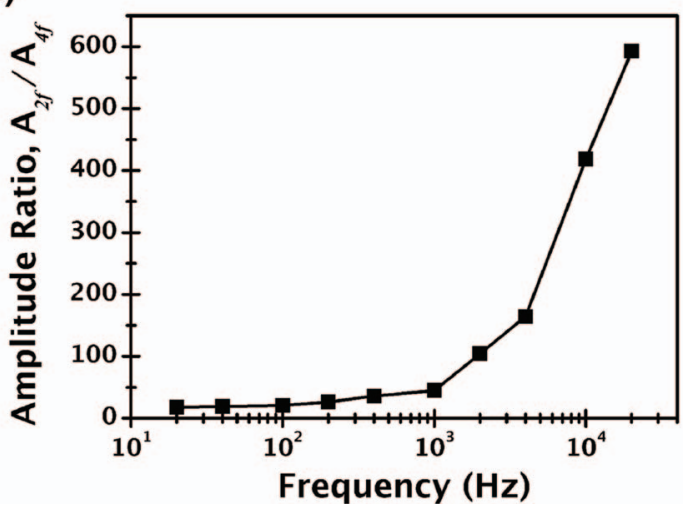

FIG. 7. (a) Thermoreflectance amplitude at $20 \mathrm{~Hz}, 200 \mathrm{~Hz}, 2 \mathrm{kHz}$, and $20 \mathrm{kHz}$ modulation with $17 \mathrm{~V}_{\mathrm{pp}}$ total excitation as a function of the frequency normalized by the modulation frequency showing dominant even harmonics. (b) Thermoreflectance amplitude ratio of $2 f$ to $4 f$ as a function of the modulation frequency.

gate time at various frequencies when the cantilever is operated with $8.5 \mathrm{~V}$ for $\mathrm{DC}$ heating and $17 \mathrm{~V}_{\mathrm{pp}}$ for $\mathrm{AC}$ heating. Thermoreflectance signals were acquired at a sampling rate of $10 \mathrm{~Hz}$ for $1 \mathrm{~h}$. At each condition, the thermoreflectance resolution improves as the gate time increases to $\sim 1 \mathrm{~s}$. However, the resolution deteriorates as the gate time further increases beyond $1 \mathrm{~s}$. The resolution generally improves as the modulation frequency increases. In case of $20 \mathrm{kHz}$, the best thermore-

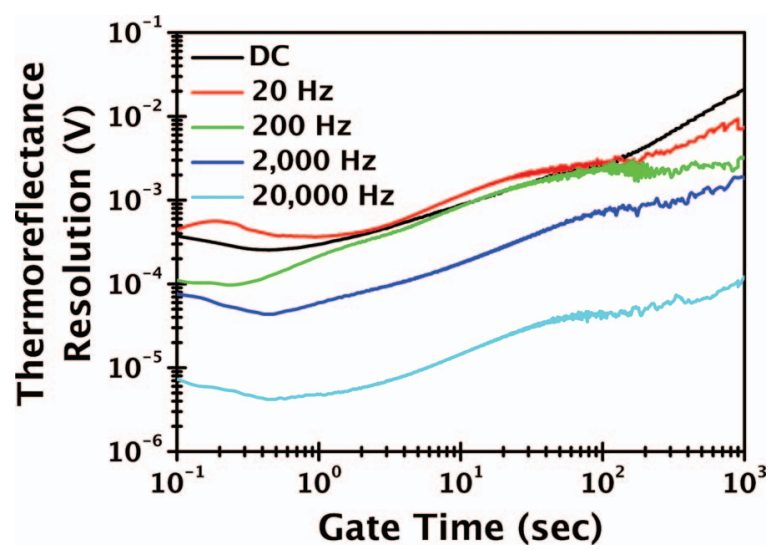

FIG. 8. Thermoreflectance signal resolutions based on Allan variance as a function of the gate time for DC, $20 \mathrm{~Hz}, 200 \mathrm{~Hz}, 2 \mathrm{kHz}$, and $20 \mathrm{kHz}$ modulation with $8.5 \mathrm{~V}$ for $\mathrm{DC}$ and $17 \mathrm{~V}_{\mathrm{pp}} \mathrm{AC}$ heating, respectively. The voltage unit for $\mathrm{AC}$ operation is $\mathrm{V}_{\mathrm{rms}}$. flectance is $4.2 \mu \mathrm{V}_{\mathrm{rms}}$ that corresponds to $8.0 \mathrm{mK}_{\mathrm{rms}}$. It should be noted that most uncertainty in the thermoreflectance thermometry is originated from its calibration using the Raman spectroscopy. A better calibration needs to be developed to avoid such systematic uncertainty in future.

\section{CONCLUSIONS}

We developed DC and AC thermal characterization techniques for heated microcantilevers by measuring and analyzing thermoreflectance signals at various input powers and frequencies. Thermoreflectance signals exhibit an oscillatory feature characterized by local minimum and maximum points, which sensitively change with the power dissipation. This result confirms that the thermoreflectance strongly depends on the device thickness when a thin device is to be measured. Through the AC thermoreflectance measurement at various modulation frequencies, we demonstrated that the thermoreflectance thermometry can measure the temperature oscillation with in-phase and out-of-phase components due to its superior temporal resolution. This capability was applied to the heated microcantilever, revealing that the AC temperature shrinks to the heater region while its amplitude decreases as the modulation frequency increases, and the thermal response of the heated microcantilever becomes more out of phase when the cantilever is operated beyond its thermal cut-off frequency, i.e., $\sim 1 \mathrm{kHz}$. The frequency-domain finite element analysis also verifies the unique capability of the thermoreflectance thermometry in measuring the AC temperature at high frequencies. Although the heated microcantilever was used as a proof of concept, the obtained results could be generally applicable to characterization of various thermal microelectromechanical systems devices.

\section{ACKNOWLEDGMENTS}

This research was supported by the Basic Science Research Program through the National Research Foundation of Korea (NRF), funded by the Ministry of Education, Science and Technology (2011-0012942). K.P. also thanks the financial support of NSF (CBET 1067441) for the frequencydomain finite element analysis of the heated microcantilever.

${ }^{1}$ J. Lee, T. Beechem, T. L. Wright, B. A. Nelson, S. Graham, and W. P. King, J. Microelectromech. Syst. 15, 1644 (2006).

${ }^{2}$ H. J. Mamin, Appl. Phys. Lett. 69, 433 (1996).

${ }^{3}$ P. E. Sheehan, L. J. Whitman, W. P. King, and B. A. Nelson, Appl. Phys. Lett. 85, 1589 (2004).

${ }^{4}$ B. A. Nelson, W. P. King, A. R. Laracuente, P. E. Sheehan, and L. J. Whitman, Appl. Phys. Lett. 88, 033104 (2006).

${ }^{5}$ E. O. Sunden, T. L. Wright, J. Lee, W. P. King, and S. Graham, Appl. Phys. Lett. 88, 033107 (2006).

${ }^{6}$ O. Fenwick, L. Bozec, D. Gredgington, A. Hammiche, G. M. Lazzerini, Y. R. Silberberg, and F. Cacialli, Nat. Nanotechnol. 4, 664 (2009).

${ }^{7}$ D. Pires, J. L. Hedrick, A. D. Silva, J. Frommer, B. Gotsman, H. Wolf, M. Despont, U. Duerig, and A. W. Knoll, Science 328, 732 (2010).

${ }^{8}$ F. Yang, E. Wornyo, K. Gall, and W. P. King, Nanotechnology 18, 285302 (2007).

${ }^{9}$ B. Gotsmann and U. Durig, Langmuir 20, 1495 (2004).

${ }^{10}$ J. Duvigneau, H. Schonherr, and G. J. Vancso, ACS Nano 4, 6932 (2010).

${ }^{11}$ O. S. Ovchinikova, M. Nikiforov, S. Jesse, J. A. Bradshaw, and G. J. V. Berkel, ACS Nano 5, 5526 (2011). 
${ }^{12}$ S. Jesse, M. P. Nikiforov, L. T. Germinario, and S. V. Kalinin, Appl. Phys. Lett. 93, 073104 (2008).

${ }^{13}$ J. Lee, A. Liao, E. Pop, and W. P. King, Nano Lett. 9, 1356 (2009).

${ }^{14}$ Z. Q. Wei, D. B. Wang, S. Kim, S. Y. Kim, Y. K. Hu, M. K. Yakes, A. R. Laracuente, Z. T. Dai, S. R. Marder, C. Berger, W. P. King, W. A. de Heer, P. E. Sheehan, and E. Riedo, Science 328, 1373 (2010).

${ }^{15}$ B. Kwon, M. Rosenberger, R. Bhargava, D. G. Cahill, and W. P. King, Rev. Sci. Instrum. 83, 015003 (2012).

${ }^{16}$ B. Mitra and A. Gaitas, Rev. Sci. Instrum. 80, 023703 (2009).

${ }^{17}$ K. Park, J. Lee, Z. M. Zhang, and W. P. King, Rev. Sci. Instrum. 78, 043709 (2007).

${ }^{18}$ B. A. Nelson and W. P. King, Rev. Sci. Instrum. 78, 023702 (2007).

${ }^{19}$ B. Lee and W. P. King, Rev. Sci. Instrum. 83, 074902 (2012).

${ }^{20}$ K. Park, J. Lee, Z. M. M. Zhang, and W. P. King, J. Microelectromech. Syst. 16, 213 (2007).

${ }^{21}$ U. Durig, J. Appl. Phys. 98, 044906 (2005).

${ }^{22}$ B. W. Chui, T. D. Stowe, Y. S. Ju, K. E. Goodson, T. W. Kenny, H. J. Mamin, B. D. Terris, R. P. Ried, and D. Rugar, J. Microelectromech. Syst. 7, 69 (1998).

${ }^{23}$ M. R. Abel, T. L. Wright, W. P. King, and S. Graham, IEEE Trans. Compon. Packag. Technol. 30, 200 (2007).

${ }^{24}$ B. A. Nelson and W. P. King, Sens. Actuators, A 140, 51 (2007).
${ }^{25}$ T. Beechem, S. Graham, S. P. Kearney, L. M. Phinney, and J. R. Serrano, Rev. Sci. Instrum. 78, 061301 (2007).

${ }^{26}$ Y. S. Ju and K. E. Goodson, J. Heat Transfer 120, 306 (1998).

${ }^{27}$ S. Grauby, A. Salhi, J. M. Rampnoux, H. Michel, W. Claeys, and S. Dilhaire, Rev. Sci. Instrum. 78, 074902 (2007).

${ }^{28}$ S. Grauby, A. Salhi, L. D. P. Lopez, W. Claeys, B. Charlot, and S. Dilhaire, Microelectron. Reliab. 48, 204 (2008).

${ }^{29}$ A. Soni and S. B. Wen, J. Phys. D: Appl. Phys. 45, 185101 (2012).

${ }^{30} \mathrm{G}$. Tessier, G. Jerosolimski, S. Hole, D. Fournier, and C. Filloy, Rev. Sci. Instrum. 74, 495 (2003).

${ }^{31}$ B. W. Chui, M. Asheghi, Y. S. Ju, K. E. Goodson, T. W. Kenny, and H. J. Mamin, Microscale Thermophys. Eng. 3, 217 (1999).

${ }^{32}$ G. E. Jellison and F. A. Modine, J. Appl. Phys. 76, 3758 (1994).

${ }^{33}$ B. J. Lee, Z. M. Zhang, E. A. Early, D. P. DeWitt, and B. K. Tsai, J. Thermophys. Heat Transfer 19, 558 (2005).

${ }^{34}$ See supplementary material at http://dx.doi.org/10.1063/1.4797621 for explaining the reason why there are dark regions in AC thermoreflectance maps.

${ }^{35}$ S. Reggiani, M. Valdinoci, L. Colalongo, M. Rudan, G. Baccarani, A. D. Stricker, F. Illien, N. Felber, W. Fichtner, and L. Zullino, IEEE Trans. Electron Devices 49, 490 (2002).

${ }^{36}$ W. Kuzmicz, Solid-State Electron. 29, 1223 (1986). 